\title{
A Kinetic Model of Tumor/immune System Cellular Interactions
}

\author{
LUISA ARLOTTI ${ }^{\mathrm{a}, *}$, ANDREA GAMBA ${ }^{\mathrm{b}}$ and MIROSLAW LACHOWICZ \\ ${ }^{\mathrm{a}}$ Department of Civil Engineering, University of Udine, via delle Scienze, 208, 33100 Udine, Italy; ${ }^{\mathrm{b}}$ Department of Mathematics, Politecnico di Torino, \\ Corso Duca degli Abruzzi, 24, 10129 Turin, Italy; ${ }^{\mathrm{c}}$ Department of Mathematics, Warsaw University, Banacha 2, 02097 Warsaw, Poland
}

(Received 1 August 2000; In final form 23 April 2001)

\begin{abstract}
In this paper, a model of cellular tumor dynamics in competition with the immune system is proposed. The characteristic scale of the phenomenon is the cellular one and the model is developed with probabilistic methods analogous to those of the kinetic theory. The interacting individuals are the cells of the populations involved in the competition between the tumor and the immune system. Interactions can change the activation state of the tumor and cause tumor proliferation or destruction. The model is expressed in terms of a bi-linear system of integro-differential equations. Some preliminary mathematical analysis of the model as well as computational simulations are presented.
\end{abstract}

Keywords: Cellular dynamics; Immune system; Tumor cells; Kinetic models; Non-linear dynamics

\section{INTRODUCTION}

Immunologists have recently shown an increasing interest in mathematical modeling of tumor dynamics in competition with the immune system, possibly under the action of external medical treatment. Models can simulate medical treatment and support choosing and applying suitable therapeutic strategies. Mathematical modeling in medicine and immunology has a long tradition and has known important successes. It can promote both improvement of clinical therapies and developing of mathematical theories. For instance, the therapy based on the mathematical work by Lasota et al. (1981) was successfully applied on patients by Ważewska-Czyżewska (1981).

The reader is addressed to review papers devoted to modeling, analysis, numerical simulations and control of tumor/immune system interactions. In particular, we mention the review papers by Bellomo and De Angelis (1998) and Bellomo and Preziosi (2000), which provide a rather complete description of the state of the art, and the review papers by Chaplain (1996), Byrne (1999), Owen and Sherratt (1999) and Please et al. (1999), devoted to the various macroscopic stages of evolution of the tumor. For general bibliography, see also the special issue (Chaplain, 1999).
The evolution of a neoplastic cell (see e.g. Herberman, 1982 ) is regulated by the genes contained in its nucleus. Genes can be either activated or suppressed when signals stimulate receptors on the cell surface and are then transmitted to the cell nucleus. In extreme situations, particular signals can induce a cell to reproduce itself in the form of identical descendants (the so-called clonal expansion) or to die (the so-called apoptosis, or programmed death). When clonal expansion appears, a competition between tumor cells and the immune system is activated. If the immune system is active and able to recognize the tumor cells, it may be able to develop destruction mechanisms, possibly resulting in the destruction of the aggressive host. Otherwise, tumor growth may develop progressively.

Summarizing, the evolution of neoplastic cells can be described according to the following steps:

1. loss of differentiation and replication: the cells start to reproduce in the form of identical descendants (clonal expansion);

2. interaction (activation or inhibition) and competition at the cellular level with immune and environmental cells, e.g. through the emission of cytokine signals;

3. condensation of tumor cells, macroscopic diffusion and angiogenesis;

*Corresponding author. E-mail: arlotti@dic.uniud.it 
4. detachment of metastases and propagation.

The first two steps imply cellular and sub-cellular interactions, while the last two can be described at the macroscopic level.

In this paper, we propose a model for the interaction at the cellular level.

Mathematical models of cellular phenomena related to the interaction between tumor cells and the immune system have been developed by Bellomo et al. (1996), Firmani et al. (1999) and Arlotti et al. (2000) using methods which are typical of kinetic theory. Such models are stated in terms of systems of integro-differential equations (like Boltzmann kinetic equation- $c f$. Ferziger and Kaper, 1972), with quadratic non-linearities, describing the evolution of the densities of cell populations with respect to the cell activation state. These models take into account various interactions between cell populations, such as conservative interactions, which modify the cells' activation state but preserve the number of cells, and proliferative-destructive interactions which modify the number of cells.

In the paper by Arlotti et al. (2000), a class of models of population dynamics with kinetic interactions taking into account transitions among different populations was proposed. These models are generalizations of the Jäger and Segel (1992) kinetic model.

In the model (2.11) which we propose here, we describe a simulation of the immune response of the organism through production of both active immune cells and macrophages.

Following Arlotti et al. (2000), we start with a general class of models describing the interaction and competition of several cellular populations. Each cell is characterized by its activation state. On the other hand, each population is characterized by its size, i.e. number of cells. Both, the number densities of cell populations and the cells' activation states evolve. The evolution is determined by the interactions between pairs of cells (analogously to the kinetic theory, we take into account here, only binary interactions).

For increased readability, the details of the general mathematical framework are summarized in Appendix A. In "A model of tumor-immune system competition", we introduce our model which is described by the system of integro-differential equations (2.10). In "Numerical simulation", we report the results of numerical simulations performed on model (2.11). Some rigorous mathematical results concerning systems of the type (2.11) are exposed in Appendix B.

\section{A MODEL OF TUMOR-IMMUNE SYSTEM COMPETITION}

The general framework summarized in Appendix A is used in this section to develop a mathematical model of cellular tumor dynamics in competition with the immune system. The model is related to the first stage of tumor cell onset and proliferation, when some endothelial cells lose differentiation and start to replicate and interact with the immune system and with environmental cells. We select the cell populations, their specific activities and identify the interactions, which are significant to the evolution of the system. For each pair interaction, we first state our hypotheses in a qualitative language, then translate them in general statements about the mathematical quantities introduced in Appendix A, finally produce a sufficiently generic, analytic form for these quantities, suitable for numeric simulations.

We consider a model with a minimal number of populations: active tumor cells and immune cells. Immune cells are further subdivided into active immune cells and macrophages. The population of active immune cells is understood to be a collection of all factors produced by the immune system that can lower the activation state of tumor cells. The population of macrophages is understood to be a collection of all factors coming from the immune system that can destroy the tumor cells (we use the name "macrophages" for simplicity). The cells of the remaining populations which influence the tumor-immune system competition will be called "environmental cells". They are formally important in view of their interactions with tumor cells but they do not represent a dynamical degree of freedom, since we assume that their number and activation state is constant during the time evolution.

Summarizing, the following four populations are identified:

1. active tumor cells;

2. active immune cells;

3. macrophages;

4. environmental cells.

Here we denote conventionally with the word cell, the members of each population, for the purpose of simplicity. We associate to tumor cells also an activation state $u \in$ $[0,1]$, which is a measure of their aggressivity and proliferating ability ( $c f$. Appendix A).

With the aim of maximally simplifying the model, we assume that the only population whose internal activation state is a relevant variable is that of active tumor cells. As to the remaining populations, we neglect the presence of internal degrees of freedom and assume that the distribution functions $f_{2}, f_{3}$ and $f_{4}$ are independent of the activation states. We now proceed to the specification of the interaction structure characterizing our model (we use the notations of Appendix A).

We will assume that only the following interactions are significant:

Interactions between 1 and 2 (1-2). Interactions between active tumor and active immune cells are characterized by the following effects: 
(i) Lowering the activation state of tumor cells by active immune cells: the function $A_{1,2}^{(1)}:[0,1]^{3} \rightarrow \mathbb{R}_{+}$is such that

(1) $A_{1,2}^{(1)}=A_{1,2}^{(1)}(v, w ; u)$ is independent of $w$,

(2) $\int_{0}^{1} A_{1,2}^{(1)}(v ; u) \mathrm{d} u=1 \forall v \in[0,1]$,

(3) $A_{1,2}^{(1)}(v ; u)=0, \forall u, v \in[0,1]: v \leq u$, and the function $a_{1,2}:[0,1]^{2} \rightarrow \mathbb{R}_{+}$is such that

(4) $a_{1,2}=a_{1,2}(u, v)$ is independent of $v$,

(5) $a_{1,2} \in L_{\infty}(0,1)$,

(6) the function $(u, v) \rightarrow A_{1,2}^{(1)}(v ; u) a_{1,2}(v)$ is in $L_{\infty}$ $\left([0,1]^{2}\right)$.

Example. A representative of the class of functions specified by conditions (1)-(6), sufficiently generic for our purposes, is

with

$$
A_{1,2}^{(1)}(v ; u)=\left\{\begin{array}{cl}
2(v-u) / v^{2} & \text { for } v>u, \\
0 & \text { for } v \leq u,
\end{array}\right.
$$

$$
a_{1,2}(v)=b_{1,2} v^{2}, \quad \forall v \in[0,1]
$$

where $\mathrm{b}_{1,2}$ is a non-negative constant.

(ii) Inhibition of active immune cells by tumor cells (we assume that the fraction of inhibited immune cells is proportional to the activation state $v$ ):

$$
a_{2,1}(v, u)=b_{2,1} v, \quad \text { for } u, v \in[0,1],
$$

where $b_{2,1}$ is a non-negative constant.

Interaction between 1 and 4 (1-4). Interactions between active tumor and environmental cells are characterized by the following effects:

(i) Tumor conservative interactions. They make the tumor cells steadily progress in the activation state: the function $A_{1,4}^{1, c}:[0,1]^{3} \rightarrow \mathbb{R}_{+}$is such that

(1) $A_{1,4}^{1, c}=A_{1,4}^{1, c}(v, w ; u)$ is independent of $w$,

(2) $\int_{0}^{1} A_{1,4}^{1, c}(v ; u) \mathrm{d} u=1 \forall v \in[0,1]$,

(3) $A_{1,4}^{1, c}(v ; u)=0, \forall u, v \in[0,1]: u \leq v$, and the function $a_{1,4}:[0,1]^{2} \rightarrow \mathbb{R}_{+}$is such that

(4) $a_{1,4}=a_{1,4}(u, v)$ is independent of $v$,

(5) $a_{1,4} \in L_{\infty}(0,1)$,

(6) the function $(u, v) \rightarrow A_{1,4}^{1, c}(v ; u) a_{1,4}(v)$ is in $L_{\infty}$ $\left([0,1]^{2}\right)$.

Example. A representative of the class of functions specified by conditions (1)-(6), sufficiently generic for our purposes, is

$$
A_{1,4}^{1, c}(v ; u)=\left\{\begin{array}{cc}
2(u-v) /(1-v)^{2} & \text { for } u>v \\
0 & \text { for } u \leq v
\end{array}\right.
$$

and

$$
a_{1,4}(v)=b_{1,4}(1-v)^{2}, \quad \forall v \in[0,1],
$$

where $\mathrm{b}_{1,4}$ is a non-negative constant.

(ii) The distribution function $f_{4}$ of environmental cells is assumed to be constant during the evolution. We normalize the number of cells of each population to the number of environmental cells, thus

$$
f_{4}=n_{4}=1
$$

(iii) tumor proliferative interactions. They trigger the production of new tumor cells with a rate proportional to $1-u$ (the probability of producing new tumor cells with small activation state should be higher than that with high activation state) and to the average activation of tumor $\int_{0}^{1} v f_{1}(t, v) \mathrm{d} v$. Therefore we assume: the function $A_{1,4}^{1, p}$ : $[0,1]^{3} \rightarrow \mathbb{R}_{+}$is such that

(1) $A_{1,4}^{1, p}=A_{1,4}^{1, p}(v, w ; u)$ is independent of $w$,

(2) $\int_{0}^{1} A_{1,4}^{1, p}(v ; u) \mathrm{d} u \geq 0 \forall v \in[0,1]$,

(3) $\int_{\rho}^{1} A_{1,4}^{1, p}(v ; u) a_{1,4}(v) f_{1}(t, v) \mathrm{d} v=b_{1,4}^{(1)}(1-u)$ $\int_{0}^{\mathrm{P}} v f_{1}(t, v) \mathrm{d} v, \forall v \in[0,1]$, where $b_{1,4}^{(1)}$ is a nonnegative constant.

Example. A representative of the class of functions specified by conditions (1)-(3), sufficiently generic for our purposes, is

$$
A_{1,4}^{1, p}(v ; u)=\frac{b_{1,4}^{(1)}(1-u) v}{b_{1,4}(1-v)^{2}} .
$$

Finally, we may define

$$
A_{1,4}^{(1)}=A_{1,4}^{1, c}+A_{1,4}^{1, p} .
$$

(iv) Stimulation of immune response (production of both active immune cells and macrophages). For $i=2,3$ we assume the function $A_{1,4}^{(i)}:[0,1]^{3} \rightarrow \mathbb{R}_{+}$is such that

(1) $A_{1,4}^{(i)}=A_{1,4}^{(i)}(v, w ; u)$ is independent of $w$,

(2) $\int_{0}^{1} A_{14}^{(i)}(v ; u) \mathrm{d} u \geq 0 \forall v \in[0,1]$,

(3) $\int_{0}^{1} A_{1,4}^{(i)}(v ; u) a_{1,4}(v) f_{1}(t, v) \mathrm{d} v=b_{1,4}^{(i)} \int_{0}^{1} f_{1}(t, v) \mathrm{d} v$, $\forall u \in[0,1]$, where $b_{1,4}^{(i)}$ is a non-negative constant.

Example. A representative of the class of functions specified by conditions (1-3), sufficiently generic for our purposes, is

$$
A_{1,4}^{(i)}(v, u)=\frac{b_{1,4}^{(i)}}{b_{1,4}} \frac{1}{(1-v)^{2}} .
$$

Interaction between 1 and 3 (1-3). Interactions between active tumor cells and macrophages are characterized by the following effects: 
i) They destroy a fraction of the tumor cells. We assume that $a_{1,3}=b_{1,3}$ is a non-negative constant.

ii) The tumor cells inhibit macrophages (we assume that the rate of interaction is proportional to the activation state $v$ ):

$$
a_{3,1}(u, v)=b_{3,1} v, \quad \text { for } u, v \in[0,1]
$$

where $b_{3,1}$ is a non-negative constant.

Interaction between $i$ and $4(i-4, i=2,3)$. In some particular cases, the processes of controlling the production of active immune cells and macrophages can be additionally included. We assume that $a_{2,4}=b_{2,4}$ and $a_{3,4}=b_{3,4}$ are non-negative constants. They contribute to linear death terms.

All the remaining (not mentioned above) $A_{j, k}^{i}$ and $a_{j, k}$ - see Appendix A-are assumed to be zero. In some particular cases, some of the above mentioned parameters of the model (i.e. the constants $\left.b_{j, k}, b_{j, k}^{(i)}\right)$ may be equal to 0 .

These interactions define a phenomenological description of cell interaction and competition ruled by subcellular mechanisms (like emission-reception of cytokine signals, etc.).

Finally, we assume that an inlet of tumor cells is possible and described by a function $\mathscr{S}=\mathscr{S}(u)$ such that $\mathscr{S}$ is a smooth decreasing function of $u \in[0,1]$ and $\mathscr{S}(1)=0$.

We point out that the parameters characterizing the model are the following:

- $b_{1,2}$ - characterizing the depression of tumor activation due to the encounter with immune cells;

- $b_{1,4}$-characterizing the steady progress of tumor cells towards increasing activation state;

- $b_{1.4}^{(1)}$-characterizing the proliferative activity of tumor cells;

- $b_{1,3}$-characterizing the destruction of tumor cells by macrophages;

- $b_{1,4}^{(2)}$-characterizing the immune response of the organism, triggered by the presence of tumor cells;

- $b_{2,1}$-characterizing the inhibition of production of immune cells due to the encounter with tumor cells;

- $b_{2,4}$-characterizing the inhibition of production of immune cells by environmental cells;

- $b_{1,4}^{(3)}$-characterizing the production of macrophages, triggered by the presence of tumor cells;

- $b_{3,1}$ - characterizing the inhibition of production of macrophages due to the encounter with active tumor cells;

- $b_{3,4}$-characterizing the inhibition of production of macrophages by environmental cells;

- the function $\mathscr{S}_{1}$-characterizing the inlet of tumor cells.
We are now ready to write down explicitly the evolution system related to the above defined model,

$$
\begin{aligned}
\frac{\partial f_{1}}{\partial t}(t, u)= & b_{1,2} n_{2}(t) \times 2 \int_{u}^{1}(v-u) f_{1}(t, v) \mathrm{d} v \\
& -u^{2} f_{1}(t, u)+b_{1,4} 2 \int_{0}^{u}(u \\
& -v) f_{1}(t, v) \mathrm{d} v-(1-u)^{2} f_{1}(t, u) \\
& +b_{1,4}^{(1)}(1-u) \int_{0}^{1} v f_{1}(t, v) \mathrm{d} v \\
& -b_{1,3} n_{3}(t) f_{1}(t, u)+S_{1}(u),
\end{aligned}
$$

$$
\begin{aligned}
\frac{\mathrm{d} n_{2}}{\mathrm{~d} t}(t)= & b_{1,4}^{(2)} \int_{0}^{1} f_{1}(t, v) \mathrm{d} v-b_{2,1} n_{2}(t) \int_{0}^{1} v f_{1}(t, v) \mathrm{d} v \\
& -b_{2,4} n_{2}(t), \\
\frac{\mathrm{d} n_{3}}{\mathrm{~d} t}(t)= & b_{1,4}^{(3)} \int_{0}^{1} f_{1}(t, v) \mathrm{d} v-b_{3,1} n_{3}(t) \int_{0}^{1} v f_{1}(t, v) \mathrm{d} v \\
& -b_{3,4} n_{3}(t) .
\end{aligned}
$$

We recall that the relevant dynamical variables are the distribution function $f_{1}(t, u)$ of tumor cells with respect to their activation, and the overall densities $n_{2}, n_{3}$ of immune cells and macrophages. A general theory for the systems of the type (2.11) is presented in Appendix B.

\section{NUMERICAL SIMULATION}

We simulated the Cauchy problem for the integrodifferential model (2.11) using an adaptive Runge-Kutta method. Integrals were performed using a GaussChebyshev quadrature formula with 11 nodes.

As an initial condition, we assume the presence of a small population of low-activity tumor cells (created in earlier times by some event whose nature we do not further investigate). We do not consider a continuous inlet of tumor cells, thus choosing $S_{1}(u)=0$.

By simulating the time evolution of the model (2.11), one observes that tumor cells proliferate and progress in the activity scale. After a (possibly long) period of dormancy, there appears a vast population of highly activated tumor cells, which triggers the reaction of the immune system. The response of the immune system can show three essentially different behaviors:

i) it can be sufficiently activated to destroy the tumor cells;

ii) the reaction can be sufficient to stop the uncontrollable proliferation of the tumor cells, without being able to completely destroy them; 



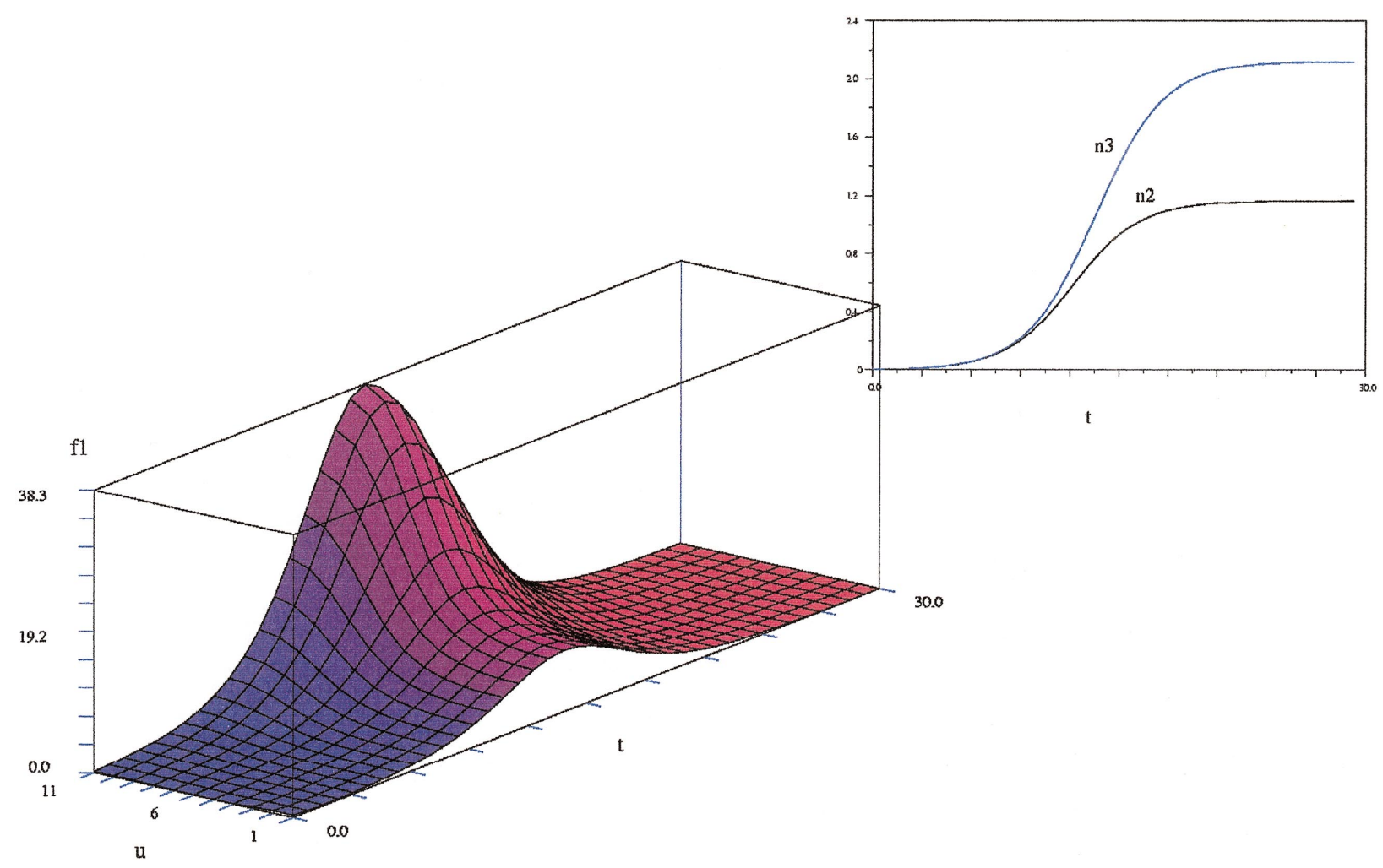

FIGURE 3 A scenario similar to that of Fig. 2, observed on a longer time scale $\left(b_{1,3}=0.3\right)$.

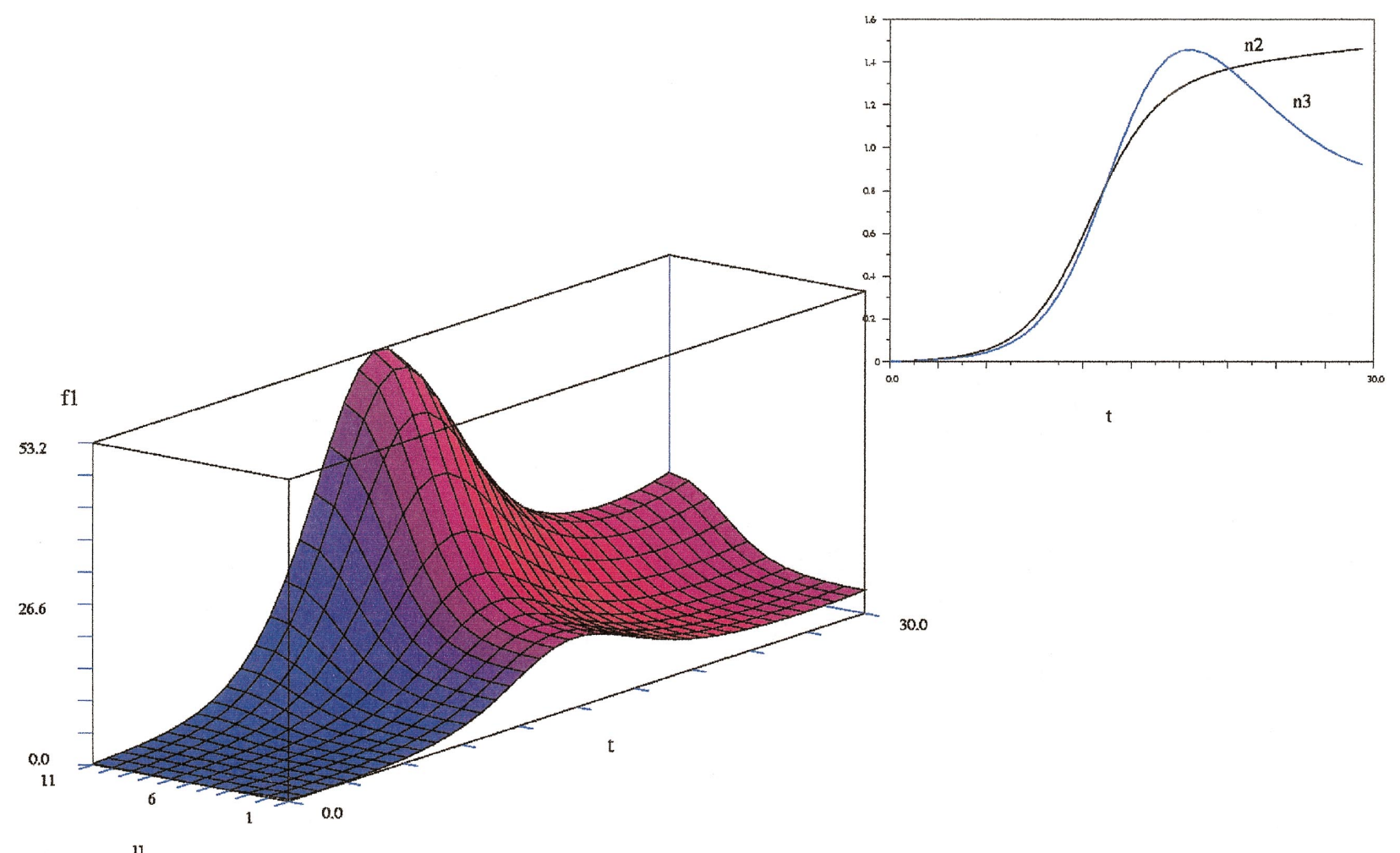

FIGURE 4 After the first reaction to tumor proliferation, the production of macrophages is relaxed, allowing a renewal of tumor aggression $\left(b_{3,4}=0.1\right)$. 


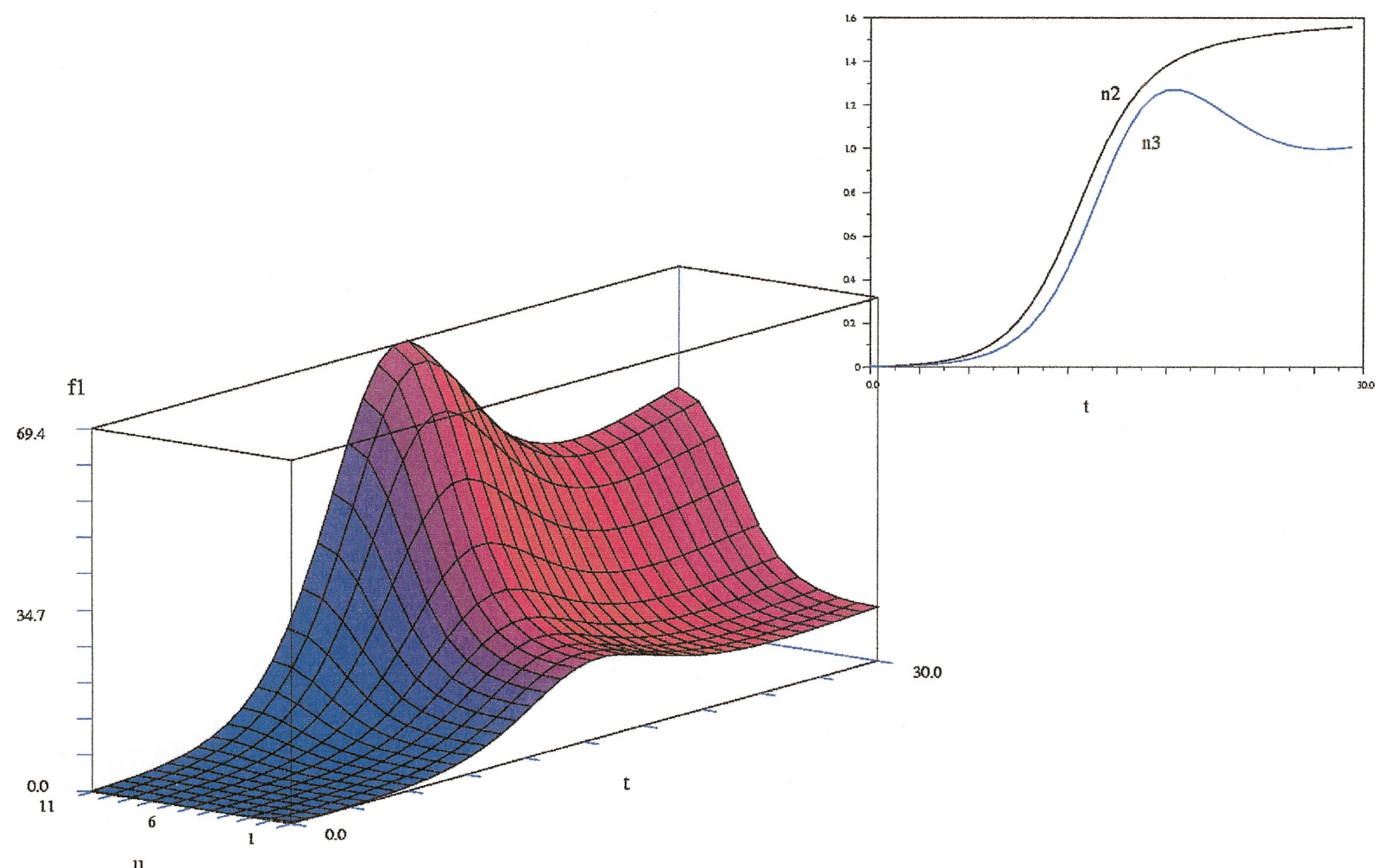

FIGURE 5 The same effect as in Fig. 4, with more intensity $\left(b_{3,4}=0.2\right)$.

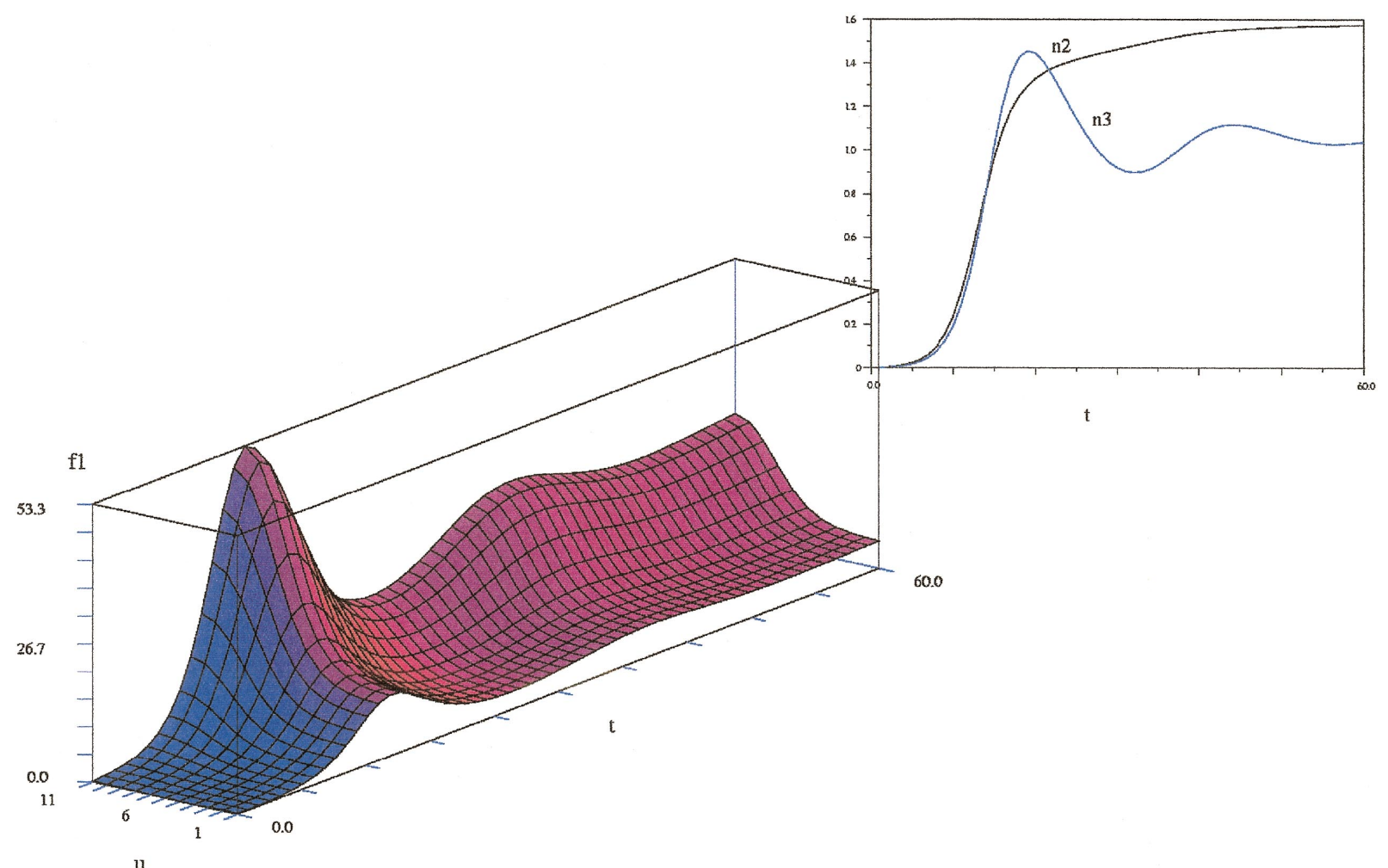

FIGURE 6 The same evolution as in Fig. 4, on a longer time scale $\left(m_{3,4}=0.1\right)$. One notices the oscillating behavior of the tumor-immune competition. 
iii) the reaction is unable to stop tumor evolution.

We observe that one of the parameters of (2.11) fixes the time scale and can be chosen to be equal to 1 without any loss of generality: let it be $b_{1,4}$, which describes the steady progress of tumor cells in the activity scale.

We report here the results of the exploration of the subspace of parameters characterized by $b_{1,4}^{(3)}=b_{1,4}^{(2)}=$ 0.01 . These values give the time-scale of the response of the immune system. In principle, the values $b_{1,4}^{(3)}$ and $b_{1,4}^{(2)}$ are different and give rise to two different time scales, but at the moment, we see no physical reason to introduce this complication and we have assumed for simplicity that they are equal. The small value 0.01 accounts for the comparatively slow response of the immune system and could of course be varied.

We assign a fixed value also to $b_{1,2}$ (action of the immune cells) and proceed to vary $b_{1,3}$ (destruction of tumor cells by macrophages). In the first sequence of simulations, we do not introduce death factors, so that $b_{2,4}=0, b_{3,4}=0$.

The result of the simulations is visualized as a 3-D plot of $f_{1}$ against the activity $u$ and time $t$ using false colors indicating the degree of activation of the immune system (blue for no activation, red for strong activation-the choice of the specific color scale changes from figure to figure). The time evolution of the $n_{2}$ and $n_{3}$ densities (immune cells and macrophages) is shown in the insets. The time scale in all of the plots is conventional, and having fixed $b_{1,4}=1$, we can think of the time unit as proportional to the natural rate of progression of the tumor cells in the activity scale. Ticks on the $u$ axis identify the nodes of the quadrature method.

By varying $b_{1,3}$, one observes several regimes.

1. In Fig. 1, we see an uncontrollable growth of the tumor cells, which cannot be stopped by the insufficient reaction of the immune system $\left(b_{1,3}=0.01\right)$.

2. In Fig. 2, the prompt reaction of the immune system is able to cope with tumor proliferation $\left(b_{1,3}=5\right)$.

3. In Fig. 3, we observe a similar scenario on a longer time scale (30 conventional units; $b_{1,3}=0.3$ ).

We then pass to analyze the effect of death factors in the model (2.11). With the introduction of death factors, immune cells and macrophages do not accumulate without limit, but act on a well defined (statistic) time scale and are then destroyed.

4. In Fig. 4 after the first reaction to tumor proliferation, we observe a relaxation in the production of macrophages, allowing an incipient renewal of the tumor aggression $\left(b_{3,4}=0.1\right)$.

5. In Fig. 5, the same effect is observed with more intensity $\left(b_{3,4}=0.2\right)$.

6. In Fig. 6, we observe the same evolution as in Fig. 4, but on a longer time scale $\left(b_{3,4}=0.1\right)$. A series of oscillations in the tumor-immune competition is clearly noticeable. This kind of behavior corresponds to a separatrix in the parameter space, dividing regions where tumor proliferation is uncontrasted and regions where tumor cells are efficiently destroyed by the immune system. In practice, the numerical simulation produces a finite number of oscillations, which, depending on the choice of the point in parameter space, eventually lead either to the proliferating behavior or to a situation in which the immune system prevails. The presence of such oscillations is realistic and experimentally confirmed.

\section{CONCLUSIONS}

We introduced a simple model of tumor-immune system competition inspired by kinetic theory and described by the system of integro-differential equations (2.11). We analyzed the abstract mathematical problem posed by (2.11), obtaining the existence result exposed in Appendix B. We simulated the Cauchy problem for model (2.11) using an adaptive Runge-Kutta method and a GaussChebyshev quadrature formulae.

As a result of the present investigation, we observe that the model presents a wealth of behaviors that can hopefully be compared with experimental data on in vitro tumor cell evolution. In dependence of the values of the parameters, one observes either an uncontrollable growth of the tumor population, or an efficient reaction of the immune system which is able to control tumor proliferation. As a separation between these two extreme situations, one observes prolonged antagonistic behavior between the tumor cells and the immune system. This scenario, which emerges from a (by necessity) partial investigation of the space of parameters, is encouraging and prompts for future work of comparison with experimental data.

\section{Acknowledgements}

The present work was partially supported by EC Programme "Human Potential—Research Training Networks" No. RTN1-1999-00294, "Using Mathematical Modelling and Computer Simulation to Improve Cancer Therapy". The Authors wish to express their sincere gratitude to Prof. Nicola Bellomo and Prof. Luigi Preziosi for their advices and criticism.

\section{References}

Arlotti, L., Bellomo, N. and Lachowicz, M. (2000) "Kinetic equations modelling population dynamics", Transport Theory and Statistical Physics 29(1/2), 125-139.

Bellomo, N. and De Angelis, E. (1998) "Strategies of applied mathematics towards an immuno-mathematical theory on tumors and immune system interactions", Mathematical Models and Methods in Applied Sciences 8, 1403-1429.

Bellomo, N. and Preziosi, L. (2000) "Modelling and mathematical problems related to tumor evolution and its interaction with the 
immune system", Mathematical and Computer Modelling 32 , 413-452.

Bellomo, N., Preziosi, L. and Forni, G. (1996) "Tumor immune system interaction: the kinetic cellular theory", In: Adam, J. and Bellomo, N., eds, A Survey of Models on Tumor Immune Systems Dynamics (Birkhäuser, Boston), pp 135-186.

Bremermann, H.J. (1983) "Parasites at the origin of life", Journal of Mathematical Biology 16, 165-180.

Byrne, H.M. (1999) "A comparison of the roles of localized and non localized growth factors in solid tumor growth", Mathematical Models and Methods in Applied Sciences 9, 541-568.

Capasso, V. (1996) Mathematical Structures of Epidemic System (Springer, New York).

Chaplain, M. (1996) "Avascular growth, angiogenesis and vascular growth in solid tumors: the mathematical modelling of stages of tumor developments", Mathematical Computation and Modelling 23, 47-88.

Chaplain, M. (1999) "Special issue on mathematical models for the growth, development and treatment of tumors", Mathematical Models and Methods in Applied Sciences 9, 4.

Ferziger, J.K. and Kaper, H.G. (1972) Mathematical Theory of Transport Processes in Gases (North-Holland, Amsterdam).

Firmani, B., Guerri, L. and Preziosi, L. (1999) "Tumor/immune system competition with medically induced activation/deactivation", Mathematical Models and Methods in Applied Sciences 9, 491-512.

Foryś, U. (1995) "Interleukin mathematical model of an immune system", Journal of Biological Systems 3, 889-902.

Hastings, S. and Murray, J. (1975) "The existence of oscillatory solutions in the Feld-Noyes model for the Belousov-Zhabotinskii reaction", SIAM Journal of Applied Mathematics 28, 678-688.

Herberman, R.B. (1982) NK Cells and Other Natural Effector Cells (Academic Press, New York).

Jäger, E. and Segel, L. (1992) "On the distribution of dominance in a population of interacting anonymous organisms", SIAM Journal of Applied Mathematics 52, 1442-1468.

Kirschner, D. and Panetta, J.C. (1998) "Modeling immunotherapy of the tumor-immune interaction", Journal of Mathematical Biology 37, $235-252$.

Lasota, A., Mackey, M.C. and Ważewska-Czyżewska, M. (1981) "Minimizing therapeutically induced anemia", Journal of Mathematical Biology 13, 149-158.

Marchuk, G.I. (1983) Mathematical Models in Immunology (Publication Division of Optimization Software INC, New York).

Owen, M.R. and Sherratt, J.A. (1999) "Mathematical modelling of macrophage dynamics in tumors", Mathematical Models and Methods in Applied Sciences 9, 513-539.

Please, C.P., Pettet, G.J. and McElwain, D.L.S. (1999) "A vascular tumor dynamics and necrosis", Mathematical Models and Methods in Applied Sciences 9, 569-579.

Smoller, J. (1983) Shock Waves and Reaction-Diffusion Equations (Springer, New York).

Stout, R.D. and Suttles, J. (1995) T-Cell Signaling of Macrophage Activation: Cell Contact-Dependent and Cytokine Signals (Springer, Berlin).

Tomlison, I. and Boomer, W. (1995) "Failure of programmed cell death and differentiation as causes of tumors: some simple mathematical models", Proceedings of the National Academy of Sciences of the USA 92, 11130-11134.

Ważewska-Czyżewska, M. (1981) Erythrokinetics (Medical Publications, Warsaw).

\section{APPENDIX A - GENERAL FRAMEWORK}

We start from the following set of assumptions:

(AA.1) The system is constituted by $d \geq 1$ interacting cell populations.

(AA.2) Each cell is characterized by its activation state $u \in \mathscr{U}$ (in this paper, for simplicity, we assume that $\mathscr{U}=$ [0,1], but $\mathscr{U}$ can be a more complicated set).
(AA.3) The function $f_{i}=f_{i}(t, u), f_{i}:[0, \infty[\times[0,1] \rightarrow$ $\mathbb{R}_{+}(i=1, \ldots, d)$ defines the density of cells of the $i$ th population with activation state $u$ at time $t \geq 0$. The number of cells of the $i$ th population at time $t \in[0, \infty[$ is given by

$$
n_{i}(t)=\int_{0}^{1} f_{i}(t, u) \mathrm{d} u
$$

and the total number of cells at time $t \in[0, \infty$ [ is given by

$$
N(t)=\sum_{i=1}^{d} n_{i}(t)
$$

(AA.4) Interactions between pairs of cells are homogeneous in space and instantaneous (without time delay). They may change the activation state of cells as well as the population size by shifting cells into another activation state or by destroying or creating cells. Only binary interactions are taken into account.

(AA.5) The rate of interaction between the cells of the $j$ th population with activation state $u \in[0,1]$ and the cells of the $k$ th population with activation state $v \in[0,1]$ $(j, k=\ldots, d)$ is given by the function

$$
a_{j, k}=a_{j, k}(u, v), \quad a_{j, k}:[0,1]^{2} \rightarrow \mathbb{R}_{+}, \quad j, k=1, \ldots, d .
$$

(AA.6) The transition into the $i$ th population with activation state $u \in[0,1]$ due to the interaction of cells of the $j$ th population with activation state $v \in[0,1]$ with cells of the $k$ th population with activation state $w \in[0,1]$ is described by the function

$$
\begin{aligned}
& A_{j, k}^{(i)}=A_{j, k}^{(i)}(v, w ; u), \quad A_{j, k}^{(i)}:[0,1]^{3} \rightarrow \mathbb{R}_{+}, \\
& \quad i, j, k=1, \ldots, d .
\end{aligned}
$$

The class of models considered in Arlotti et al. (2000) is the following:

$$
\begin{aligned}
& \frac{\partial f_{i}}{\partial t}(t, u)=G_{i}[\mathbf{f}](t, u)-L_{i}[\mathbf{f}](t, u)+S_{i}(t, u), \\
& u \in[0,1] \quad i=1, \ldots, d
\end{aligned}
$$

where $\mathbf{f}=\left\{f_{i}\right\}_{i=1}^{d} ; G_{i}$ is the gain term due to binary interactions which drive cells into the $i$ th population and into the activation state $u$,

$G_{i}[\mathbf{f}](u)=\sum_{j, k=1}^{d} \int_{0}^{1} \int_{0}^{1} A_{j, k}^{(i)}(v, w ; u) a_{j, k}(v, w) f_{j}(v) f_{k}(w) \mathrm{d} v \mathrm{~d} w$

$L_{i}$ is the loss term due to binary interactions which drive cells out of the $i$ th population or out of the activation state $u$,

$$
L_{i}[\mathbf{f}](u)=f_{i}(u) \sum_{j=1}^{d} \int_{0}^{1} a_{i, j}(u, v) f_{j}(v) \mathrm{d} v,
$$


$S_{i}$ is the production or migration term of cells of the $i$ th population with activation state $u$ due to any artificial inlet.

The above framework can be generalized in order to include spatial diffusion processes and interactions with time delay. Various versions of Eq. (A1) were analyzed in Arlotti et al. (2000) and Bellomo et al. (1996) (see also references therein).

Remark A1. Note that, if for all $i, j, k=1, \ldots, d$, the functions $a_{(j, k)}, A_{j, k}^{(i)}$ and $S_{i}$ are constants, Eq. (A1) takes the simpler form of a system of ordinary differential equations:

$$
\begin{aligned}
\frac{\mathrm{d} n_{i}}{\mathrm{~d} t} & =\sum_{j, k=1}^{d} A_{j, k}^{(i)} a_{j, k} n_{j} n_{k}-n_{i} \sum_{k=1}^{d} a_{i, k} n_{k}-S_{i}, \\
i & =1, \ldots, d
\end{aligned}
$$

The particular choices of parameters of the system (A2) result in various important examples of models known in the literature, such as Bremermann's (1983) model of phage-host dynamics, Kermack-McKendrick (SIR) model (see Capasso (1996)), Marchuk's model of infections diseases (Marchuk, 1983) with zero time delay. If spatial diffusion terms or time delay are included then, as examples one recovers the Field-Noyes model for the Belousov-Zhabotinsky reactions in chemical kinetics (Hastings and Murray, 1975; Smoller, 1983) or Marchuk's model with time delay (Marchuk, 1983; Foryś, 1995).

Referring to models with a zero source term $S_{i}=0$ (for $i=1, \ldots, d)$, the following classification was proposed in Arlotti et al. (2000):

- Conservative case:

$$
\begin{aligned}
& \sum_{i=1}^{d} \int_{0}^{1} A_{j, k}^{(i)}(v, w ; u) \mathrm{d} u=1, \quad \forall j, k=1, \ldots, d, \\
& \quad \forall v, w \in[0,1] .
\end{aligned}
$$

In this case, the total number of cells $N(t)$ is preserved, $N(t)=N(0), t>0$.

- Proliferative case:

$$
\begin{aligned}
& \sum_{i=1}^{d} \int_{0}^{1} A_{j, k}^{(i)}(v, w ; u) \mathrm{d} u>1, \quad \forall j, k=1, \ldots, d, \\
& \quad \forall v, w \in[0,1] .
\end{aligned}
$$

In this case, the total number of cells $N(t)$ increases: $t \uparrow \Rightarrow N(t) \uparrow$.

- Destructive case:

$$
\begin{aligned}
& \sum_{i=1}^{d} \int_{0}^{1} A_{j, k}^{(i)}(v, w ; u) \mathrm{d} u<1, \quad \forall j, k=1, \ldots, n, \\
& \quad \forall v, w \in[0,1] .
\end{aligned}
$$

In this case, the total number of cells $N(t)$ decreases: $t \uparrow \Rightarrow N(t) \downarrow$.

In some cases, one can consider the class of models in which the distribution function $f_{i}\left(i=d_{1}, \ldots, d, 1 \leq d_{1} \leq\right.$ $d$ ) of the last $d-d_{1}+1$ populations are independent of the activation state $u$,

$$
\begin{gathered}
f_{i}(t, u)=n_{i}(t), \quad \forall u \in[0,1], \quad t \geq 0, \\
i=d_{1} \ldots, d .
\end{gathered}
$$

More precisely: we assume that

(S.1) $a_{i, j}=a_{i, j}(u, v)$ is independent of $v$ for all $i=$ $1, \ldots, d_{1}-1$ and $j=d_{1}, \ldots, d$;

(S.2) $a_{i, j}=a_{i, j}(u, v)$ is independent of $u$ and $v$ for all $i=d_{1}, \ldots, d$ and $j=d_{1}, \ldots, d$;

(S.3) $a_{i, j}=a_{i, j}(u, v)$ is independent of $u$ for all $i=$ $d_{1}, \ldots, d$ and $j=1, \ldots, d_{1}-1$;

(S.4) $A_{j, k}^{(i)} a_{j, k}=A_{j, k}^{(i)}(v, w ; u) a_{j, k}(v, w)$ is independent of $w$ for all $i=1, \ldots, d_{1}-1, j=d_{1}, \ldots, d$ and $k=1, \ldots, d_{1}-$ 1 ;

(S.5) $A_{j, k}^{(i)}=A_{j, k}^{(i)}(v, w ; u)$ is independent of $v$ and $w$ for all $i=1, \ldots, d$ and $j, k=d_{1}, \ldots, d$;

(S.6) $A_{j, k}^{(i)}(v, w ; u)$ is independent of $w$ for all $i=$ $1, \ldots, d, j=1, \ldots, d_{1}-1$ and $k=d_{1}, \ldots, d$;

(S.7) $A_{j, k}^{(i)}(v, w ; u)$ is independent of $v$ for all $i=$ $d_{1}, \ldots, d, j=d_{1} \ldots, d$ and $k=1, \ldots, d_{1}-1$;

(S.8) $S_{i}(t, u)$ is independent of $u$ for all $i=d_{1} \ldots, d$.

Under these assumptions the system of evolution equation reads

$$
\begin{aligned}
& \frac{\partial f_{i}}{\partial t}(t, u)=\sum_{j, k=1}^{d_{1}-1} \int_{0}^{1} \int_{0}^{1} A_{j, k}^{(i)}(v, w ; u) a_{j, k}(v, w) \\
& \quad \times f_{j}(t, v) f_{k}(t, w) \mathrm{d} v \mathrm{~d} w \\
& \quad+\sum_{j=1}^{d_{1}-1} \sum_{k=d_{1}}^{d} n_{k}(t) \int_{0}^{1} \alpha_{j, k}^{(i)}(v ; u) f_{j}(t, v) \mathrm{d} v \\
& \quad+\sum_{k, j=d_{1}}^{d} \beta_{j, k}^{(i)}(u) n_{j}(t) n_{\mathrm{k}}(t)-f_{i}(t, u) \\
& \quad \times \sum_{j=1}^{d_{1}-1} \int_{0}^{1} a_{i, j}(u, v) f_{j}(t, v) \mathrm{d} v+\sum_{j=d_{1}}^{d} a_{i, j}(u) n_{j}(t) \\
& \quad+S_{i}(t, u), \quad i=1, \ldots, d_{1}
\end{aligned}
$$




$$
\begin{aligned}
& \frac{\mathrm{d} n_{i}}{\mathrm{~d} t}(t)=\sum_{j, k=d_{1}}^{d} \tilde{\beta}_{j, k}^{(i)} n_{j}(t) n_{k}(t) \\
& +\sum_{j=1}^{d_{1}-1} \sum_{k=d_{1}}^{d} n_{k}(t) \int_{0}^{1} \tilde{\alpha}_{j, k}^{(i)}(v) f_{j}(t, v) \mathrm{d} v \\
& +\sum_{j, k=1}^{d_{1}-1} \int_{0}^{1} \int_{0}^{1} \tilde{A}_{j, k}^{(i)}(v, w) a_{j, k}(v, w) \\
& \quad \times f_{j}(t, v) f_{k}(t, w) \mathrm{d} v \mathrm{~d} w \\
& \quad-n_{i}(t) \sum_{j=1}^{d_{1}-1} \int_{0}^{1} a_{i, j}(v) f_{j}(t, v) \mathrm{d} v+\sum_{j=d_{1}}^{d} a_{i, j} n_{j}(t) \\
& +S_{i}(t), \quad i=d_{1}, \ldots, d,
\end{aligned}
$$

where

$$
\begin{gathered}
\alpha_{j, k}^{(i)}(v, u)=\int_{0}^{1} A_{j, k}^{(i)}(v, w ; u) a_{j, k}(v, w) \mathrm{d} w \\
+\int_{0}^{1} A_{k, j}^{(i)}(w, v ; u) a_{k, j}(w, v) \mathrm{d} w \\
\beta_{j, k}^{(i)}(u)=\int_{0}^{1} \int_{0}^{1} A_{j, k}^{(i)}(v, w ; u) a_{j, k}(v, w) \mathrm{d} v \mathrm{~d} w \\
\tilde{\beta}_{j, k}^{(i)}=\int_{0}^{1} \beta_{j, k}^{(i)}(u) \mathrm{d} u, \tilde{\alpha}_{j, k}^{(i)}(v)=\int_{0}^{1} \alpha_{j, k}^{(i)}(v ; u) \mathrm{d} u, \tilde{A}_{j, k}^{(i)}(v, w) \\
=\int_{0}^{1} A_{j, k}^{(i)}(v, w ; u) \mathrm{d} u .
\end{gathered}
$$

\section{APPENDIX B - MATHEMATICAL PROBLEMS}

In this appendix, we provide information on the qualitative behavior of the solution of the initial value problem for the abstract version of the system (2.11).

The abstract version of the model is expressed in terms of the following integro-differential bi-linear system of equations:

$$
\begin{aligned}
\frac{\partial f_{1}}{\partial t}(t, u)= & n_{2}(t) \mathscr{B}_{1,2}\left[f_{1}\right](t, u)+\mathscr{B}_{1,4}\left[f_{1}\right](t, u) \\
& +B(u) \int_{0}^{1} v f_{1}(t, v) \mathrm{d} v-b_{1,3} n_{3}(t) f_{1}(t, u) \\
& +S_{1}(u), \\
\frac{\mathrm{d} n_{i}}{\mathrm{~d} t}(t)= & b_{1,4}^{(i)} \int_{0}^{1} f_{1}(t, v) \mathrm{d} v \\
& -b_{i, 1} n_{i}(t) \int_{0}^{1} v f_{1}(t, v) \mathrm{d} v-b_{i, 4} n_{i}(t), \\
i= & 2,3,
\end{aligned}
$$

where $\mathscr{B}_{1, i}, i=2,4$, are linear operators such that

$$
\begin{aligned}
\mathscr{B}_{1, i}[f](u)= & \int_{0}^{1} A_{1, i}(u, v) a_{1, i}(v) f(v) \mathrm{d} v \\
& -a_{1, i}(u) f(u),
\end{aligned}
$$

$(u, v) \rightarrow A_{1, i}(u, v) a_{1, i}(v)$ and $a_{1, i}$ are smooth nonnegative functions on $[0,1]^{2}$ and $[0,1]$, respectively,

$$
\begin{gathered}
\int_{0}^{1} A_{1, i}(u, v) \mathrm{d} u=1 \quad \forall v \in[0,1], \\
A_{1,2}(u, v)=0, \quad \forall u, v \in[0,1]: v \leq u, \\
A_{1,4}(u, v)=0, \forall u, v \in[0,1]: u \leq v,
\end{gathered}
$$

$\mathscr{B}_{1,2}$ describes the lowering tumor activation state due to interactions with immune cells, whereas $\mathscr{B}_{1,4}$ does the raising tumor activation state due to interactions with environmental cells;

$B$ is a smooth function that characterizes the proliferative activity of tumor cells; $b_{1,3}, b_{1,4}^{(i)}, b_{i, 1}$, and $b_{i, 4}(i=2,3)$ are non-negative constants introduced in Appendix A; $S_{1}$ is a smooth non-negative function characterizing the inlet of tumor cells.

Let $L_{1}(0,1)$ be the Lebesgue space of measurable, realvalued functions which are integrable on $[0,1]$. The norm is denoted by $\|\cdot\|_{1}$.

Let $H_{m, 1}(0,1)$ be the Sobolev space of functions which, together with all their derivatives of order $k \leq m$, are in $L_{1}$ $(0,1)$. The norm is denoted by $\|\cdot\|_{m, 1}$.

Let

$$
\begin{aligned}
\mathbf{X} & =\left\{\mathbf{f}=\left(f_{1}, n_{2}, n_{3}\right): f_{1} \in L_{1}(0,1),\left|n_{2}\right|<\infty,\left|n_{3}\right|\right. \\
& <\infty\}
\end{aligned}
$$

be the Banach space equipped with the norm $\||\mathbf{f}|\| \mid=$ $\left\|f_{1}\right\|_{1}+\left|n_{2}\right|+\left|n_{3}\right|$.

Let

$\mathbf{X}^{+}=\left\{\mathbf{f}=\left(f_{1}, n_{2}, n_{3}\right) \in \boldsymbol{X}: f_{1} \geq 0, n_{2} \geq 0, n_{3} \geq 0\right\}$.

Finally, let

$$
\begin{aligned}
\mathbf{X}_{m} & =\left\{\mathbf{f}=\left(f_{1}, n_{2}, n_{3}\right):\right. \\
& \left.f_{1} \in H_{m, 1}(0,1),\left|n_{2}\right|<\infty,\left|n_{3}\right|<\infty\right\}
\end{aligned}
$$

be the Banach space equipped with the norm $\|\mid f\|_{m}=$ $\left\|f_{1}\right\|_{m, 1}+\left|n_{2}\right|+\left|n_{3}\right|$.

Theorem B1. Let $S_{1} \in L_{1}(0,1), S_{1} \geq 0$ a.e., and $\mathbf{f}_{0} \in$ $\mathbf{X}^{+}$. For every $t_{0}>0$ there exists a unique solution

$$
\mathbf{f} \in C^{0}\left(\left[0, t_{0}\right] ; \mathbf{X}\right) \cap C^{1}(] 0, t_{0}[; \mathbf{X})
$$


of Eq. (B1) with the initial datum $\mathbf{f}_{0}$. The solution $\mathbf{f}$ satisfies

$$
\mathbf{f}(t) \in \mathbf{X}^{+}, \quad t \in\left[0, t_{0}\right],
$$

and

$$
\sup _{\left[0, t_{0}\right]}\||\mathbf{f}|\| \leq \mathrm{c}_{t_{0}}\left(\left\|\left|\mathbf{f}_{(0)}\right|\right\|+\left\|S_{1}\right\|_{1}\right),
$$

for some constant $c_{t_{0}}$ (depending on $t_{0}$ ).

Moreover, if $\mathbf{f}_{0} \in \mathbf{X}_{\mathrm{m}}$, for some $m \geq 1$, then $\mathbf{f}(t) \in \mathbf{X}_{m}$, for $t \in\left[0, t_{0}\right]$.

Proof. Note that the solution to Eq. (B1) is a priori nonnegative. Applying (B2)-(B5) and integrating both sides of the first equation of (B1) with respect to the variable $u$, we see that no contribution comes from the two first rows, which describe conservative interactions. Therefore

$$
\begin{aligned}
& \frac{\mathrm{d}}{\mathrm{d} t} n_{1}(t) \leq \bar{B} n_{1}(t)+\bar{S}, \quad \frac{\mathrm{d}}{\mathrm{d} t} n_{i}(t) \leq b_{1,4}^{(i)} n_{1}(t), \\
& \quad i=2,3,
\end{aligned}
$$

where $n_{1}(t)=\left\|f_{1}(t)\right\|_{1}, \bar{B}=\|B\|_{1}$ and $\bar{S}=\left\|S_{1}\right\|_{1}$. Thus the non-linear system (B1) "behaves not worse" than a linear system.

Remark B2. The solution $\mathbf{f}$ of Eq. (B1) satisfies

$$
\begin{aligned}
n_{1}(t) & \leq n_{1}(0) \exp \left(\bar{B} t-b_{1,3} \int_{0}^{t} n_{3}\left(t_{1}\right) \mathrm{d} t_{1}\right) \\
+ & \bar{S} \int_{0}^{t} \exp \left(\frac{b_{1,4}^{(1)}}{2}\left(t-t_{1}\right)-b_{1,3} \int_{t_{1}}^{t} n_{3}\left(t_{2}\right) \mathrm{d} t_{2}\right) \mathrm{d} t_{1}, \\
\forall t & \geq 0,
\end{aligned}
$$

where the notation of the proof of Theorem B1 is used. If $b_{i, 1}>0$ then

$$
\begin{aligned}
n_{i}(t) \geq & \left(n_{i}(0)-\frac{b_{1,4}^{(i)}}{b_{i, 1}}\right) \exp \left(-b_{i, 4} t-b_{i, 1} \int_{0}^{t} n_{1}\left(t_{1}\right) \mathrm{d} t_{1}\right) \\
& +\frac{b_{1,4}^{(i)}}{b_{i, 1}}-\frac{b_{1,4}^{(i)} b_{i, 4}}{b_{i, 1}} \\
& \times \int_{0}^{t} \exp \left(-b_{i, 4}\left(t-t_{1}\right)-b_{i, 1} \int_{t_{1}}^{t} n_{1}\left(t_{2}\right) \mathrm{d} t_{2}\right) \mathrm{d} t_{1}, \\
\forall t>0 &
\end{aligned}
$$

for $i=2,3$.
By Remark B2, we obtain

Corollary B3. Let $b_{3,1}>0$, and $b_{3,4}=0$.

i) If

$$
n_{3}(0) \geq \frac{b_{1,4}^{(3)}}{b_{3,1}}
$$

then

$$
n_{3}(t) \geq \frac{b_{1,4}^{(3)}}{b_{3,1}}, \quad \forall t \geq 0 .
$$

ii) If (B12a) and

$$
\frac{b_{1,4}^{(1)}}{2} \leq \frac{b_{1,3} b_{1,4}^{(3)}}{b_{3,1}}
$$

are satisfied then

$$
n_{1}(t) \leq n_{1}(0)+\bar{S}, \quad \forall t \geq 0 .
$$

iii) If (B12a) and (B13a) and one of the following two inequalities

$$
n_{3}(0)>\frac{b_{1,4}^{(3)}}{b_{3,1}}
$$

or

$$
\frac{b_{1,4}^{(1)}}{2}<\frac{b_{1,3} b_{1,4}^{(3)}}{b_{3,1}}
$$

together with $S_{1} \equiv 0$ be satisfied, then

$$
\lim _{t \rightarrow \infty} n_{1}(t)=0 .
$$

Theorem B1 delivers the (global in time) existence, uniqueness and regularity of solutions to Eq. (B1). Corollary B3 expresses the conditions for the extinction of active tumor cells. Note that Eq. (2.11) is a particular case of Eq. (B1) and hence the results of Appendix B can be directly applied to Eq. (2.11). 


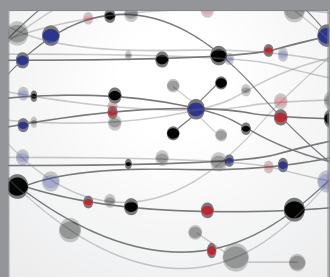

The Scientific World Journal
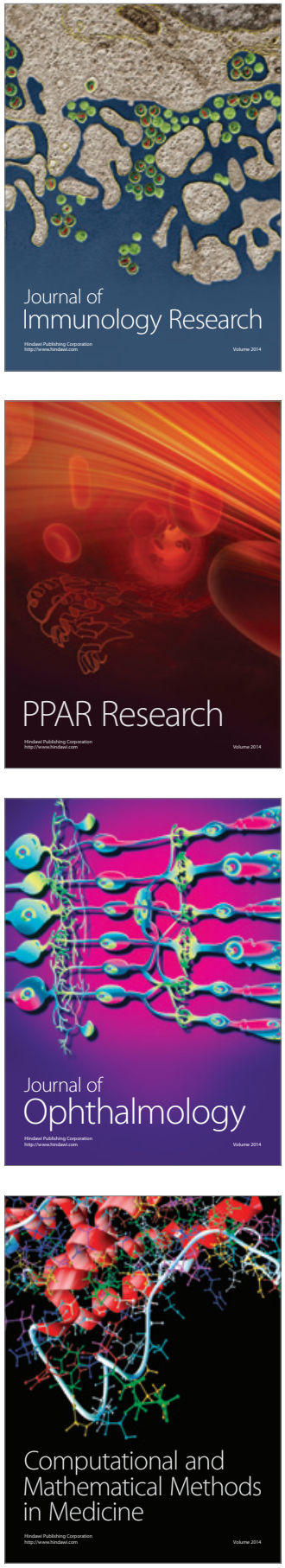

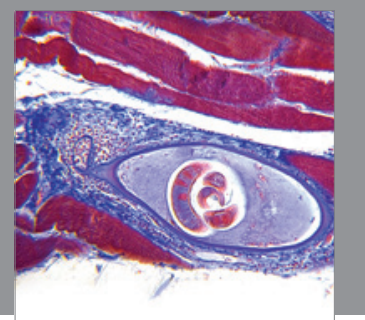

Gastroenterology

Research and Practice
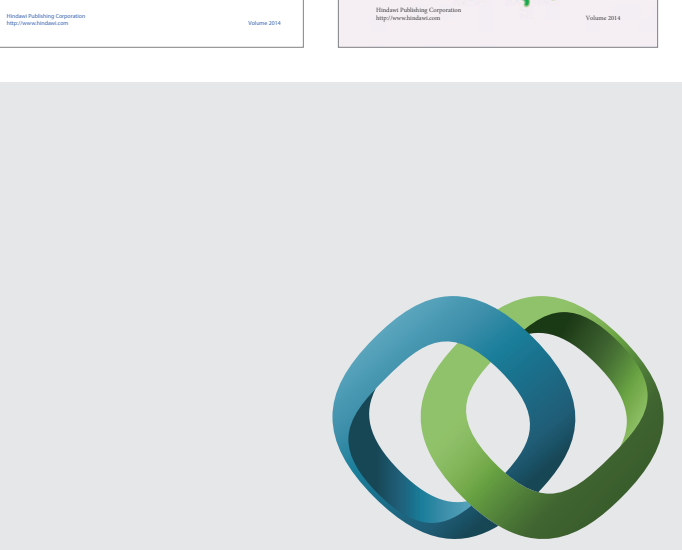

\section{Hindawi}

Submit your manuscripts at

http://www.hindawi.com
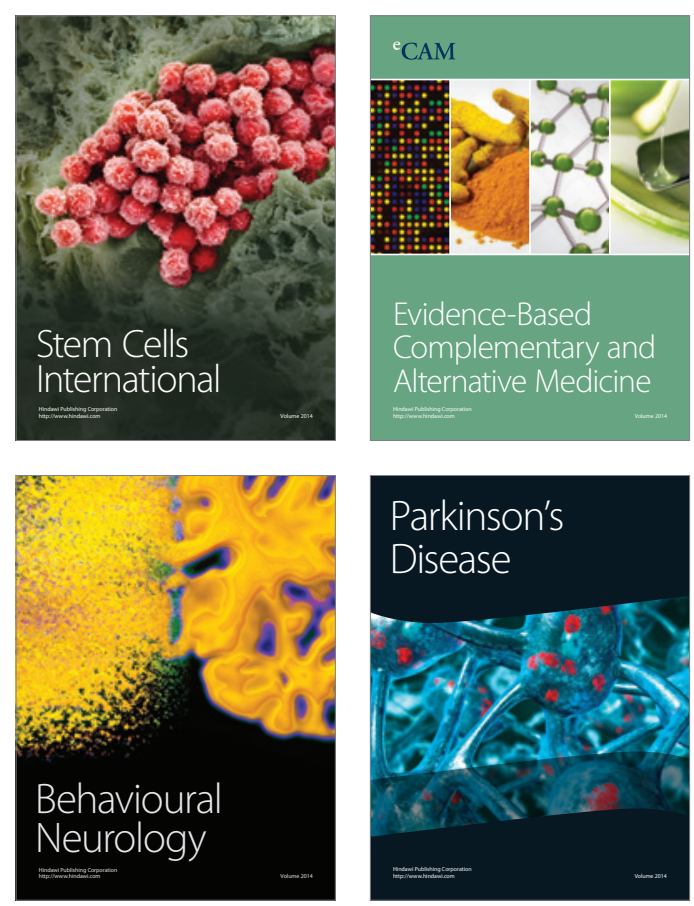

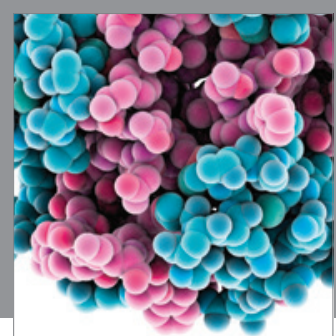

Journal of
Diabetes Research

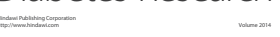

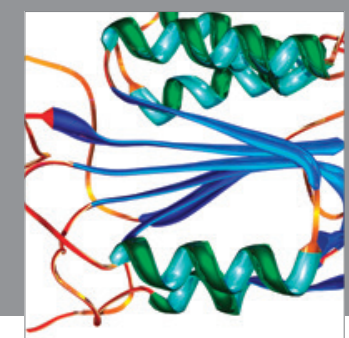

Disease Markers
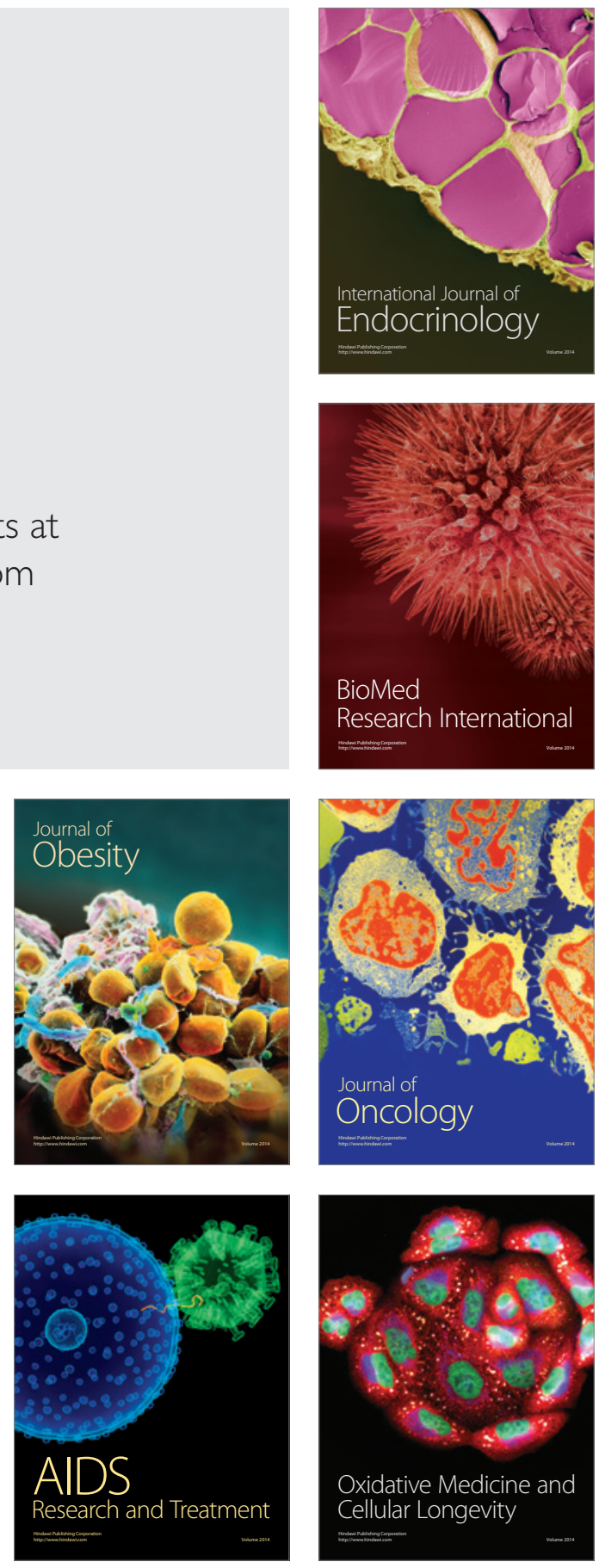